\title{
A retrospective Cohort Study to Predict Acute Kidney Injury Based on the Coagulation and Inflammation in ICU Patients with Sepsis
}

\section{Kunmei Lai}

First Affiliated Hospital of Fujian Medical University

\section{Zhenhuan Zou}

First Affiliated Hospital of Fujian Medical University

\section{Xiongjian Xiao}

First Affiliated Hospital of Fujian Medical University

\section{Yueteng Xu}

First Affiliated Hospital of Fujian Medical University

\section{Chen Zhang}

First Affiliated Hospital of Fujian Medical University

\section{Caiming Chen}

First Affiliated Hospital of Fujian Medical University

\section{Yujia Wang}

First Affiliated Hospital of Fujian Medical University

\section{Yinshuang Li}

First Affiliated Hospital of Fujian Medical University Jiandong Lin

First Affiliated Hospital of Fujian Medical University Yanfang Xu ( $\nabla$ xuyanfang99@hotmail.com)

First Affiliated Hospital of Fujian Medical University https://orcid.org/0000-0002-6207-545X

\section{Research article}

Keywords: sepsis, acute kidney injury, clinical prediction, coagulation, inflammation

Posted Date: February 9th, 2021

DOI: https://doi.org/10.21203/rs.3.rs-194895/v1

License: (1) (1) This work is licensed under a Creative Commons Attribution 4.0 International License. Read Full License 


\section{Abstract}

Background. Sepsis is a major cause of morbidity and mortality worldwide. Sepsis with acute kidney injury (AKI) is associated with higher mortality risk when compared with those with sepsis and without AKI. Therefore, it is necessary to detect the predictors of sepsis-associated acute kidney injury (SA-AKI) in order to timely prevent, diagnose and treat this complication.

Methods. From July 2016 to December 2019, 419 patients with sepsis admitted to the intensive care unit (ICU) were randomly divided into two groups: training group $(n=302)$ and validation group $(n=117)$. A least absolute shrinkage and selection operator (LASSO) regression was constructed to select variables within $24 \mathrm{~h}$ of admission, and then were included in a logistic regression model to find the independent risk factors of AKI. Hence, a nomogram for predicting SA-AKI with statistically significant covariates was constructed. Discrimination, calibration, and clinical utility of the nomogram performance were assessed and then validated.

Results. The risk factors yielded by logistic regression were hypertension(HT), diabetes mellitus(DM), Creactive protein(CRP), procalcitonin(PCT), activated partial thromboplastin time(APTT), platelet(PLT), and then were incorporated into the nomogram. The areas under the ROC curve of the nomogram in the training and validation groups were 0.856 and 0.885 , respectively. The calibration curves demonstrated favorable consistency between the predictions of nomogram and the actual observations in both training as well as validation groups. Decision curve analysis (DCA) showed clinical usefulness of the proposed nomogram model.

Conclusions. A risk prediction model by integrating variables can assist in identifying patients who are at high risk of developing SA-AKI. The nomogram had excellent predictive ability and might have significant clinical implications for early detection of AKI in patients undergoing sepsis.

\section{Background}

Sepsis is a life-threatening organ dysfunction caused by a dysregulated host response to infection [1]. Despite great efforts in the pathophysiological research of sepsis, early biomarkers and treatment strategies that are associated with high mortality of sepsis have been explored. However, there are still great challenges in identifying these high-risk patients [2-4]. There are nearly 250,000 cases of deaths associated with sepsis in the USA annually and nearly 37 of severe sepsis or septic shock cases of every 100 ICU admissions in China $[5,6]$. The development of sepsis is an extremely complex involving rapid pathophysiological process of systemic inflammatory network effects [7], genetic polymorphisms [8], coagulopathy [9], immune dysfunction [10], tissue damage, and abnormal responses of the host to different infectious pathogenic microorganisms and their toxins [11]. Therefore, it is important to identify high-risk patients with poor prognosis and provide timely intervention.

Multiple studies have shown that patients with sepsis and AKI have distinct characteristics of higher severity scores at admission, more non-renal organ failure, requirement of vasopressors, mechanical 
ventilation, higher mortality and longer hospital stay when compared to those with sepsis and without AKI patients [12-14]. COVID-19-associated acute kidney injury is thought to be one of the sequelae from sepsis and cytokine storm syndrome [15]. Of the 5,449 patients hospitalized with COVID-19 during March 1, 2020 to April 5, 2020 at 13 academic and community hospitals in metropolitan New York, 1,993 (36.6\%) patients have developed AKI. AKI is primarily seen in COVID-19 patients with respiratory failure, and $89.7 \%$ of patients on mechanical ventilation develop AKI showing poor prognosis [16]. Many hospitals have underestimated the problem of developing sepsis to AKI early, and critically ill patients should undergo dialysis with disease progression. SA-AKI is considered as a high risk factor of death and serious illness, and so experts in this field have called for the establishment of clinical models for predicting the occurrence of AKI [17].

Due to this, the risk prediction linked to this disease has become worthy of investigation. Several predictions were made over the past decade, wherein most of them focused on novel biomarkers. Most of the clinical prediction models were used to predict surgery-related AKI [18-21]. There is only one nomogram based on the MIMIC-III database for predicting the AKI risk estimation in patients with sepsis. However, the predictive value of this study is moderate, and the prediction performance remained unsatisfactory [22].

From the pathogenesis of SA-AKI, the coagulation abnormalities and inflammation are known to be universal symptoms in septic patients and play a key role in multiple organ dysfunction syndrome (MODS) [23-24]. Considering high mortality risk and clinical significance in patients with SA-AKI, we aimed to investigate the clinical characteristics of AKI in patients with sepsis and clinical risk factors of AKI in patients with sepsis. Also we sought to determine whether there were unique models for the prediction of SA-AKI.

\section{Methods}

\section{Subjects}

This was a single-center retrospective cohort study conducted to predict SA-AKI in patients admitted to an ICU. This was approved by the ethics committee of the First Affiliated Hospital of Fujian Medical University, which is a Tertiary Comprehensive Hospital. Patients were screened for predictor variables within $24 \mathrm{~h}$ of ICU admission. Both baseline and acute risk factors were recorded at the time of screening. From July 2016 to December 2019, 5880 critically ill patients were admitted to the ICU. Among these, 419 patients who met the criteria for Sepsis-3 (The Third International Consensus Definitions for Sepsis and Septic Shock) were enrolled in this study[1]. The exclusion criteria were as follows: (1) missing information ( $n=15)$; (2) younger than 18 years age $(n=11)$; (3) patients with stage 5 chronic kidney disease or hemodialysis within one month $(n=19)$; (4) ICU stay of less than 12 hours $(n=405)$; (5) did not meet the criteria of Sepsis-3 $(n=1621)$; and $(6)$ transferred from ICU to other departments $(n=3390)$. A flow diagram demonstrating the detailed screening process was shown in Fig. 1. 


\section{Definition of AKI}

The definition and staging of AKI were given based on the serum creatinine (Scr) levels of the Kidney Disease: Improving Global Outcomes (KDIGO) guidelines in 2012 [25]. The outcome included occurrence of AKI in patients' during hospital stay. The definition of AKI was modified by omitting urine output, which was as follows: an increase in SCr by $0.3 \mathrm{mg} / \mathrm{dL}$ within 48 hours or an increase in SCr by at least 1.5 times that of the baseline, which is known or presumed to have occurred within the 7 days.

\section{Statistical analysis}

All statistical analyses were performed using Statistical Package SPSS (version 22, SPSS Inc., Chicago, IL, USA) and R software version 3.6 (The R Foundation for Statistical Computing, www.R-project.org). All data were recorded within the first $24 \mathrm{~h}$ of admission. The measurement data were expressed as means and standard deviation (SD) or median interquartile range (IQR). Normally distributed variables were presented as means \pm standard deviation (SD), and t-test was used to compare the differences between groups. Non-normally distributed variables were presented as medians and interquartile ranges, and Mann-Whitney $U$ test was used to compare the differences between groups. Categorical data between the groups (non-AKI versus AKI) were compared by Chi-square and Fisher's exact tests. A p value of $<0.05$ was considered to be statistically significant.

Least Absolute Shrinkage and Selection Operator (LASSO) is a regression analysis method that selects predictors from a large set of variables to improve the predictive accuracy and interpretability of the selected set of predictors[26]. The regularization parameter lambda was selected based on 10-fold cross validation method and yielded minimum mean cross-validated concordance index. The predictors with nonzero coefficients in the LASSO regression model were chosen for constructing logistic regression model to find independent risk factors for AKI. A nomogram was developed for predicting SA-AKI based on independent variables according to the results of multivariable logistic regression analysis. The resulting model was first internally validated by assessing the discrimination and calibration with 1000 bootstrap resamples and calculated a relatively corrected C-index. The discriminative ability of the model was assessed by the ROC curve. ROC curves were used to illustrate the performance of a binary classifier system with varying discrimination thresholds. The goodness of fit in the calibration curve fitting was performed, which was evaluated by the Hosmer-Lemeshow test. Decision curve analysis (DCA) was conducted to determine the clinical usefulness of the nomogram by quantifying the net benefits at different threshold probabilities. A two-sided $95 \%$ confidence interval $(\mathrm{Cl})$ was constructed around the point estimate of the odd's ratio (OR). All the tests were two-sided.

\section{Results}

\section{Study cohort characteristics and outcomes}

The baseline demographics, clinical and laboratory characteristics of the whole, training, and validation groups are shown in Table 1. The present study retrospectively reviewed 419 patients with sepsis who were admitted to ICU, and were randomly divided into a training group $(n=302)$, which was used to 
develop the nomogram, and a validation group $(n=117)$, which was used to validate the nomogram. The mean age of patients was 65.45 years, and over six-tenths $(68.7 \%)$ of the patients were males. The proportions of main diagnoses, the occurrences of comorbidities, laboratory characteristics, etc. showed no significant differences $(P>0.05)$ between the training group and validation group, suggesting that the two groups are homogeneous and comparable.

\section{Table 1.Basline demographics, clinical and laboratory characteristics of patients}

\begin{tabular}{|c|c|c|c|c|}
\hline Characteristics & $\operatorname{ALL}(\mathrm{n}=419)$ & Training group(n=302) & Validation group(n=117) & $p$ value \\
\hline Age,years & $65.45( \pm 16.48)$ & $65.87( \pm 16.91)$ & $64.38( \pm 15.30)$ & 0.405 \\
\hline Gender male, $n(\%)$ & $288(68.7 \%)$ & $211(69.9 \%)$ & $77(65.8 \%)$ & 0.481 \\
\hline APACHE2 & $20.12( \pm 10.02)$ & $19.83( \pm 9.89)$ & $20.95( \pm 10.39)$ & 0.304 \\
\hline septic shock, $n(\%)$ & $305(72 \%)$ & $84(60.9 \%)$ & $221(78.6 \%)$ & 0.222 \\
\hline \multicolumn{5}{|l|}{ Infection site } \\
\hline PTPI, n(\%) & $385(91.8 \%)$ & $280(92.7 \%)$ & $105(89.7 \%)$ & 0.323 \\
\hline UTi, $n(\%)$ & $43(10.2 \%)$ & $35(11.6 \%)$ & $8(6.8 \%)$ & 0.208 \\
\hline Abdominal, $\mathrm{n}(\%)$ & $45(10.7 \%)$ & $30(9.9 \%)$ & $15(12.8 \%)$ & 0.384 \\
\hline Others, $\mathrm{n}(\%)$ & $47(11 \%)$ & $33(10.9 \%)$ & $14(12 \%)$ & 0.733 \\
\hline \multicolumn{5}{|l|}{ Comorbidity } \\
\hline $\mathrm{HT}, \mathrm{n}(\%)$ & $193(46 \%)$ & $145(48 \%)$ & $48(41 \%)$ & 0.23 \\
\hline $\mathrm{DM}, \mathrm{n}(\%)$ & $110(26.3 \%)$ & $80(26.5 \%)$ & $30(25.6 \%)$ & 0.902 \\
\hline $\mathrm{CKD}, \mathrm{n}(\%)$ & $34(8 \%)$ & $25(8.3 \%)$ & $9(7.7 \%)$ & 1 \\
\hline CLD, $n(\%)$ & $46(10 \%)$ & $32(10.6 \%)$ & $14(12.0 \%)$ & 0.728 \\
\hline $\mathrm{COPD}, \mathrm{n}(\%)$ & $46(11 \%)$ & $31(10.3 \%)$ & $15(12.8 \%)$ & 0.487 \\
\hline $\mathrm{CHF}, \mathrm{n}(\%)$ & $50(12 \%)$ & $35(11.6 \%)$ & $15(12.8 \%)$ & 0.738 \\
\hline $\mathrm{CRP}(\mathrm{mg} \mathrm{L})$ & $141.52( \pm 92.9)$ & $140.13( \pm 87.31)$ & $145.13( \pm 106.40)$ & 0.622 \\
\hline PCT (ng/ml) & $16.22(3.06-62.34)$ & $19.48[3.35,62.09]$ & $9.93[2.02,53.62]$ & 0.18 \\
\hline APTT $(S)$ & $48.47( \pm 28.1)$ & $48.00( \pm 25.64)$ & $49.71( \pm 33.95)$ & 0.578 \\
\hline $\mathrm{FB}(\mathrm{g} L)$ & $4.05( \pm 1.96)$ & $4.02( \pm 1.92)$ & $4.13( \pm 2.10)$ & 0.613 \\
\hline $\mathrm{PT}(\mathrm{S})$ & $17.56( \pm 13.3)$ & $17.47( \pm 12.06)$ & $17.78(=16.14)$ & 0.834 \\
\hline PT-INR & $1.47( \pm 0.63)$ & $1.48(=0.68)$ & $1.44( \pm 0.50)$ & 0.489 \\
\hline TT $(S)$ & $16.2[15-18.9]$ & $16.20[15.10-19.10]$ & $16.30[15.00-18.10]$ & 0.713 \\
\hline Ddimer (mgL) & $5.78[2.67-12.66]$ & $6.19[3.05-12.72]$ & $4.61[2.31-11.40]$ & 0.103 \\
\hline $\mathrm{TBL}(\mathrm{umol} / \mathrm{L})$ & $12[7.3-21.8]$ & $12.30[7.12-22.18]$ & $11.10[7.80-20.60]$ & 0.726 \\
\hline DBIL (umoll ) & $6.3[3.7-13.3]$ & $6.60[3.80-13.85]$ & $5.90[3.70-12.80]$ & 0.427 \\
\hline IBIL (umolL) & $4.9[3-8.4]$ & $4.80[2.92-8.10]$ & $5.00[3.20-8.80]$ & 0.582 \\
\hline $\mathrm{TP}(\mathrm{g} / \mathrm{L})$ & $53.45( \pm 9.32]$ & $53.14( \pm 8.94)$ & $54.34(=10.25)$ & 0.239 \\
\hline $\operatorname{ALB}(\mathrm{gL})$ & $28.55( \pm 5.59]$ & $28.40( \pm 5.44)$ & $28.94( \pm 5.98)$ & 0.377 \\
\hline $\operatorname{ALT}(\mathrm{U} / \mathrm{L})$ & $31[16-70]$ & $32.00[17.00-72.75]$ & $26.00[14.00-57.00]$ & 0.137 \\
\hline AST (U/L) & $42[23-103]$ & $44.00[24.00,103.75]$ & $34.00[21.00-85.00]$ & 0.082 \\
\hline $\mathrm{LDH}(\mathrm{U} / \mathrm{L})$ & $366[250-543]$ & $367.50[257.75-548.00]$ & $352.00[235.00-527.00]$ & 0.212 \\
\hline $\mathrm{HGB}(\mathrm{gL})$ & $96.81( \pm 24.9)$ & $96.63( \pm 23.49)$ & $97.30( \pm 28.36)$ & 0.804 \\
\hline $\operatorname{PLT}\left(10^{\prime} 9 / \mathrm{L}\right)$ & $134[65-224]$ & $136.50[69.25-220.75]$ & $118.00[52.00-225.00]$ & 0.395 \\
\hline
\end{tabular}

Table 1. All of these parameters came from the first day of ICU admission. APACHEX: acute physiology and chronic health evaluation scoring system; PTPI:

pulmonary infection; UTi:urinary infection; CKD:chronic kidney diseases; CLD:chronic liver disease; 
COPD:chronic obstructive pulmonary disease; CHF:congestive heart failure; FIB:fibrinogen;

PT:prothrombin time; INR: international normalized ratio; TT:thrombin time; TBil:total bilirubin; DBIL:direct bilirubin; IBIL: indirect bilirubin; TP:total protein; ALB:albumin; ALT: alanine aminotransferase; AST: Aspartate aminotransferase; LDH:lactate dehydrogenase;

\section{Development of a risk prediction model for AKI}

LASSO regression was implemented for training sets and 32 predictors were included in the LASSO regression analyses to predict the onset of AKI, respectively. Standardized predictors were used to compare the effects of different predictors. During model estimation, the lowest prediction error in 10 -fold cross validation was applied to select the optimal LASSO model, and finally 19 predictors were included (Fig. 2).

To determine the best predictors regarding the incidence of SA-AKI and to eliminate the multicollinearity among variables, LASSO regression was performed, and the predictors with $P$ values $<0.05$ were accepted for logistic regression. The final logistic regression included a total of 19 parameters (8 baseline characteristics, and 11 laboratory examinations). According to the $O R$ and $P$ values of $<0.05,6$ parameters were considered as independent risk factors for SA-AKI with results reported as OR $(95 \% \mathrm{CI})$, HT [2.286(1.047-5.095)], DM [2.691(1.164-6.577)], CRP [1.008(1.003-1.014)], PCT [1.013(1.0051.023)], APTT [1.035(1.008-1.067)] and PLT [0.995(0.992-0.998)].

\section{Risk of SA-AKI based on the nomogram scores}

A prognostic nomogram for early recognition of SA-AKI patients within the first $24 \mathrm{~h}$ of admission to the ICU was constructed using the multivariate logistic regression results, and points were given to the identified factors based on their regression coefficients. Several items with extreme values were excluded and considered a range of $0.05-0.95$ (Fig. 3). In the nomogram, patients with HT, DM, longer APTT, higher concentrations of CRP, PCT and lower concentrations of PLT in cycling more likely developed SA-AKI.

\section{Validation of SA-AKI nomogram}

Bootstrapping technique as qualified by Harrell's C statistic concordance index (the Harrell C-Index) was initially used to evaluate the discrimination of the model and to reduce overfitting bias. The $\mathrm{C}$-index for predicting nomogram was 0.854 through bootstrapping validation, suggesting good discrimination of the model. The nomogram demonstrated good accuracy for estimating the risk of SA-AKI in the first $24 \mathrm{~h}$, with ROC curve estimation for the risk of SA-AKI in the training and validation groups were 0.856 and 0.885 , respectively, (Fig. 4). The calibration plot was in excellent accordance between the nomogram prediction and the actual observations of SA-AKI (Fig. 5). The Hosmer-Lemeshow test of calibration had a $p$ value of 0.145 , which confirmed the goodness of fit model. 


\section{Sensitivity analyses}

The results of DCA showed clinical usefulness of the nomogram by quantifying the net benefits at different threshold probabilities. In the AKI risk nomogram, apparent performance of it showed good prediction capability (Fig. 6).

\section{Discussion}

According to the results of multivariate logistic regression analysis, HT, DM, CRP, PCT, APTT, and PLT were identified as independent predictors of SA-AKI. Clinical predictive model based on these six factors can be used for predicting the occurrence of SA-AKI, and showed high predictive power.

There are several potential explanations for these results. The most important explanations for this is related to the pathogenesis of the disease condition. Endothelial cell (ECs) play a key role in the balance between pro-inflammatory and anti-inflammatory mediators, regulating vascular tone, barrier permeability and activation of coagulation. Increased importance of pathogenic mechanisms of SA-AKI is attributed to kidney damage, resulting in a complex interaction between inflammatory cascade activation, deranged coagulation pathways and immunologic mechanisms. This in turn led to microvascular dysfunction, endothelial damage, leukocyte/platelet activation with the formation of micro-thrombi, epithelial tubular cell injury and dysfunction. ECs lose functions and switch from a quiescent to an active state and then exacerbate inflammatory response [23-24, 27-28]. Abnormalities in coagulation with concomitant inflammatory processes were closely linked to the systemic generation of thrombin and consequent formation of microthrombi, contributing to organ dysfunction [29]. Vascular injury and inflammation plays an important role in coagulation during sepsis. Simultaneously, a process called immunothrombosis, wherein platelet aggregation determines the release of cytokines and chemokines from platelet granules, leading to the recruitment of leukocytes and local inflammation [30]. Inflammation and disturbances in coagulation are inseparably tied, each acting as a positive feedback for the activation of the other [31]. Research on COVID-19 sepsis showed that during the occurrence of inflammatory reaction in the systemic organs, the microvascular system is shown to be damaged, resulting in abnormal activation of the coagulation system. This is pathologically manifested as systemic small vasculitis and extensive microthrombosis. Additionally, Professor Peng Zhiyong of the Central South Hospital and others conducted a retrospective analysis of 138 patients with COVID-19, and showed that the coagulation pathway was activated in severe COVID-19 patients due to cytokine storms, resulting in excessive consumption of coagulation factors and platelets [32]. Excessive consumption of coagulation substrate caused coagulation dysfunction, and formed a vicious circle, which led to the development of severe COVID-19 to disseminated intravascular coagulation (DIC) or multiple organ failure (MOF). Secondly, Zhipeng Xu et al have conducted a retrospective analysis on the routine coagulation indexes of 138 patients with septic shock caused by intra-abdominal infection and the 
results revealed that the plasma APTT, PT and D-dimer levels on admission to the ICU were shown to be as significant risk factors of AKI [33]. Chantalle E. Menard et al have shown that both prevalence and incidence of thrombocytopenia are high in septic shock patients. Thrombocytopenia is associated with poor prognosis[34]. Our research showed that compared with sepsis patients without AKI, patients with AKI had more anomaly inspection indicators, and this is because they demonstrated greater disturbances in blood biochemistry (e.g. CRP, PCT, APTT, and PLT). Recent studies have focused on establishing clinical prediction models for SA-AKI based on coagulation markers, but these models did not combine important factors such as inflammation indicators, and did not develop a prediction nomogram in patients with SA-AKI [14,33,35-42], and so their prediction performance is considered insufficient. Many studies have focused on the mortality of sepsis instead of identifying high-risk patients with poor prognosis early and to intervene in a timely manner [43-45]. Further studies are warranted to evaluate the advancements in machine learning techniques. Based on the pathophysiology of SA-AKI, it is necessary to establish a clinical prediction model by combining inflammation coagulation indicators to predict SAAKI.

However, there are several limitations that should be acknowledged in this study. Firstly, this was a singlecenter retrospective study, and validation of our model in other populations is required to more precisely evaluate the stability of the risk factors. Secondly, the present study was subjected to selection bias due to retrospective design. Due to limited sample size, the statistical power in comparative analyses was reduced, resulting in bias and coincidence. Some studies have shown that factors, such as IL-6, urine output and body mass index (BMI) [46-49], could influence the development and outcomes of SA-AKI might be ignored owing to the lack of complete medical records in some cases or the difficulty in data collection. Additionally, subgroup analysis of patients with mild AKI was not conducted owing to small sample size in this population. Furthermore, the time series analysis for the prediction risk of SA-AKI within the first $24 \mathrm{~h}$ of admission to the ICU was not conducted.

Despite these limitations, our study has several notable strengths. To the best of our knowledge, there were very few studies that has established the clinical prediction model of SA-AKI. Our study is the first to construct a nomogram for predicting SA-AKI based on clotting and inflammatory markers within $24 \mathrm{~h}$ of ICU admission. Although this was a single-center retrospective study, our research has important positive results and clinical significance. Traditional diagnostic indicators such as sensitivity, specificity and AUC can only measure the diagnostic accuracy of the predictive model, failing to consider the clinical utility of the specific model. Therefore, full advantage of DCA was taken and the clinical characteristics of patients were integrated into analysis. The calibration plot in our study showed excellent concordance between the nomogram prediction and the actual observations of SA-AKI, and the proposed nomogram model was shown to be clinically useful by DCA.

It is necessary to conduct further research on SA-AKI in order to identify high risk populations, employ preventive strategies, guarantee an early diagnosis and prompt and adequate treatment, which ultimately improved the patient outcomes. Our study could assist physicians in conducting early clinical interventions before the occurrence of AKI. Also sepsis stratification should be done in patients based on 
the severity of AKI. This method can form an intervention strategy based on the guidance of biomarkers to rationally use strained medical resources. Further external verification of our model should be conducted and the model should be displayed in the form of APP, web page or machine scoring to facilitate clinical practice.

\section{Conclusion}

In conclusion, a predictive model, a new nomogram, for patients with SA-AKI who were admitted to ICU has been successfully developed and validated. This risk assessment tool could help clinicians to stratify patients for primary prevention, surveillance and early therapeutic intervention, improving care and outcomes in ICU patients.

\section{Declarations}

\section{Ethics approval and consent to participate}

The Ethics Committee of the First Affiliated Hospital of Fujian Medical University approved this retrospective design and study (approval number [2018]035), and because patients' identification was not possible, the need for informed consent for this study was waived.

\section{Consent to publish}

Not applicable.

\section{Availability of data and materials}

The datasets used and/or analysed during the current study are available from the corresponding author on reasonable request.

\section{Competing interests}

The authors declare that they have no conflicts of interest.

\section{Funding}

This work was supported by National Natural Science Foundation of China (No.81870472), Joint Funds for the Innovation of Science and Technology of Fujian province (No. 2019Y9019) , Natural Science Foundation of Fujian province (No. 2020J02020) and Fujian Provincial Department of Finance (No.2020B009).

\section{Authors' Contributions}

Y.F.X, X.J.X. and J.D.L. designed the study. K.M.L., Z.H.Z and Y.F.X. wrote the first draft of the manuscript. K.M.L, Z.H.Z, X.J.X., Y.T.X, C.Z., J.Q.L., X.H. Z, and Y.S.L contributed to data collection and analysis. All 
authors approved the final version of the manuscript.

\section{References}

1. Singer M, Deutschman CS, Seymour CW, et al. The Third International Consensus Definitions for Sepsis and Septic Shock (Sepsis-3). JAMA. 2016;315(8):801-10.

2. Cavazzuti I, Serafini G, Busani S, et al. Early therapy with IgM-enriched polyclonal immunoglobulin in patients with septic shock. Intensive Care Med. 2014;40(12):1888-96.

3. Martin GS. Sepsis, severe sepsis and septic shock: changes in incidence, pathogens and outcomes. Expert Rev Anti Infect Ther. 2012;10(6):701-6.

4. Su L, Pan P, Yan P, et al. Role of vimentin in modulating immune cell apoptosis and inflammatory responses in sepsis. Sci Rep. 2019;9(1):5747.

5. GS M, DM M. S E, et al. The epidemiology of sepsis in the United States from 1979 through 2000. N Engl J Med. 2003;348(16):1546-54.

6. Moore JX, Donnelly JP, Griffin R, et al. Defining Sepsis Mortality Clusters in the United States. Crit Care Med. 2016;44(7):1380-7.

7. Balk RA. Systemic inflammatory response syndrome (SIRS): where did it come from and is it still relevant. today? Virulence. 2014;5(1):20-6.

8. Arcaroli J, Fessler MB, Abraham E. Genetic polymorphisms and sepsis. Shock. 2005;24:300-12.

9. Simmons J, Pittet JF. The coagulopathy of acute sepsis. Curr Opin Anaesthesiol. 2015;28:227-36.

10. Delano MJ, Ward PA. The immune system's role in sepsis progression, resolution, and long-term outcome. Immunol Rev. 2016;274:330-53.

11. Chen $\mathrm{H}, \mathrm{Li} Y, \mathrm{Wu} J$, et al. RIPK3 collaborates with GSDMD to drive tissue injury in lethal polymicrobial sepsis. Cell Death Differ2020;doi:10.1038/s41418-020-0524-1.

12. Bagshaw SM, Uchino S, Bellomo R, Morimatsu H, Morgera S, Schetz M, et al. Septic acute kidney injury in critically ill patients: clinical characteristics and outcomes. Clin J Am Soc Nephrol. 2007;2:431-9.

13. Uchino S, Kellum JA, Bellomo R, Doig GS, Morimatsu H, Morgera S, et al. Acute renal failure in critically ill patients: a multinational, multicenter study. JAMA. 2005;294:813-8.

14. Gameiro J, Fonseca JA, Jorge S, et al. Neutrophil, lymphocyte and platelet ratio as a predictor of mortality in septic-acute kidney injury patients. Nefrologia 2020.

15. Lubetzky M, Aull MJ, Craig-Schapiro R, et al. Kidney allograft recipients, immunosuppression, and coronavirus disease-2019: a report of consecutive cases from a New York City transplant center. Nephrol Dial Transplant. 2020. doi:10.1093/ndt/gfaa154.

16. Hirsch JS, Ng JH, Ross DW, et al. Acute Kidney Injury in Patients Hospitalized with Covid-19. Kidney Int. 2020;98(1):209-18. 
17. Cummings MJ, Baldwin MR, Abrams D, et al. Epidemiology, clinical course, and outcomes of critically ill adults with COVID-19 in New York City: a prospective cohort study. The Lancet. 2020;395(10239):1763-70.

18. Martini A, Sfakianos JP, Paulucci DJ, et al. Predicting acute kidney injury after robot-assisted partial nephrectomy: Implications for patient selection and postoperative management. Urol Oncol. 2019;37(7):445-51.

19. Martini A, Cumarasamy S, Beksac AT, et al. A Nomogram to Predict Significant Estimated Glomerular Filtration Rate Reduction After Robotic Partial Nephrectomy. Eur Urol. 2018;74(6):833-9.

20. Moulla Y, Lyros O, Adolf D, et al. A Nomogram Based on Clinical Factors to Predict the Serum Myoglobin Levels Following Bariatric Surgery. Obes Surg. 2017;28(6):1697-703.

21. Xu L, Li C, Zhao L, et al. Acute kidney injury after nephrectomy: a new nomogram to predict postoperative renal function. BMC Nephrol. 2020;21(1):181.

22. Deng F, Peng M, Li J, et al. Nomogram to predict the risk of septic acute kidney injury in the first $24 \mathrm{~h}$ of admission: an analysis of intensive care unit data. Ren Fail. 2020;42(1):428-36.

23. Fani F, Regolisti G, Delsante M, et al. Recent advances in the pathogenetic mechanisms of sepsisassociated acute kidney injury. J Nephrol. 2018;31(3):351-9.

24. Levi M, Schultz M, van der Poll T. Sepsis and thrombosis. Semin Thromb Hemost. 2013;39(5):55966.

25. Khwaja A. KDIGO clinical practice guidelines for acute kidney injury. Nephron Clin Pract. 2012;120(4):c179-184.

26. Tibshirani R. Regression shrinkage and selection via the lasso. J R Stat Soc Series B Stat Methodol. 1996;58:267-88.

27. Stasi A, Intini A, Divella C, et al. Emerging role of Lipopolysaccharide binding protein in sepsisinduced acute kidney injury. Nephrol Dial Transplant. 2017;32(1):24-31.

28. Gustot T. Multiple organ failure in sepsis: prognosis and role of systemic inflammatory response. Curr Opin Crit Care. 2011;17(2):153-9.

29. dJ MLE, vdP T. Rationale for restoration of physiological anticoagulant pathways in patients with sepsis and disseminated intravascular coagulation. Critical care medicine. 2001;29:90-4.

30. Engelmann B, Massberg S. Thrombosis as an intravascular effector of innate immunity. Nat Rev Immunol. 2013;13(1):34-45.

31. Wald R, McArthur E, Adhikari NK, et al. Changing incidence and outcomes following dialysis-requiring acute kidney injury among critically ill adults: a population-based cohort study. Am J Kidney Dis. 2015;65(6):870-7.

32. Song JC, Wang G, Zhang W, et al. Chinese expert consensus on diagnosis and treatment of coagulation dysfunction in COVID-19. Mil Med Res. 2020;7(1):19.

33. Xu Z, Cheng B, Fu S, et al. Coagulative biomarkers on admission to the ICU predict acute kidney injury and mortality in patients with septic shock caused by intra-abdominal infection. Infect Drug Resist. 
2019;12:2755-64.

34. Cabral L, Afreixo V, Almeida L, et al. The Use of Procalcitonin (PCT) for Diagnosis of Sepsis in Burn Patients: A Meta-Analysis. PLoS One. 2016;11(12):e0168475.

35. Chawla LS, Seneff MG, Nelson DR, et al. Elevated plasma concentrations of IL-6 and elevated APACHE II score predict acute kidney injury in patients with severe sepsis. Clin J Am Soc Nephrol. 2007;2(1):22-30.

36. Li D, Zhou Y, Yu J, et al. Evaluation of a novel prognostic score based on thrombosis and inflammation in patients with sepsis: a retrospective cohort study. Clin Chem Lab Med. 2018;56(7):1182-92.

37. Semeraro F, Ammollo CT, Caironi P, et al. Low D-dimer levels in sepsis: Good or bad? Thromb Res. 2019;174:13-5.

38. Souza AC, Yuen PS, Star RA. Microparticles: markers and mediators of sepsis-induced microvascular dysfunction, immunosuppression, and AKI. Kidney Int. 2015;87(6):1100-8.

39. Semeraro F, Colucci M, Caironi P, et al. Platelet Drop and Fibrinolytic Shutdown in Patients With Sepsis. Crit Care Med. 2018;46(3):e221-8.

40. Deppermann C, Kubes P. Platelets and infection. Semin Immunol. 2016;28(6):536-45.

41. Mihajlovic D, Lendak D, Mitic G, et al. Prognostic value of hemostasis-related parameters for prediction of organ dysfunction and mortality in sepsis. Turk J Med Sci. 2015;45(1):93-8.

42. Malhotra R, Kashani KB, Macedo E, et al. A risk prediction score for acute kidney injury in the intensive care unit. Nephrol Dial Transplant. 2017;32(5):814-22.

43. Naderpour Z, Momeni M, Vahidi E, et al. Procalcitonin and D-dimer for Predicting 28-Day-Mortality Rate and Sepsis Severity based on SOFA Score; A Cross-sectional Study. Bull Emerg Trauma. $2019 ; 7(4): 361-5$.

44. Inciardi RM, Adamo M, Lupi L, et al. Characteristics and outcomes of patients hospitalized for COVID19 and cardiac disease in Northern Italy. Eur Heart J. 2020;41(19):1821-9.

45. Chen T, Wu D, Chen $\mathrm{H}$, et al. Clinical characteristics of 113 deceased patients with coronavirus disease 2019: retrospective study. BMJ. 2020;368:m1091.

46. Shimazui T, Nakada TA, Tateishi Y, et al. Association between serum levels of interleukin-6 on ICU admission and subsequent outcomes in critically ill patients with acute kidney injury. BMC Nephrol. 2019;20(1):74.

47. Chawla LS, Seneff MG, Nelson DR, et al. Elevated plasma concentrations of IL-6 and elevated APACHE II score predict acute kidney injury in patients with severe sepsis. Clin J Am Soc Nephrol. 2007;2(1):22-30.

48. Jin K, Murugan R, Sileanu FE, et al. Intensive Monitoring of Urine Output Is Associated With Increased Detection of Acute Kidney Injury and Improved Outcomes. Chest. 2017;152(5):972-9.

49. Prescott HC, Chang VW, O'Brien JM Jr, et al. Obesity and 1-year outcomes in older Americans with severe sepsis. Crit Care Med. 2014;42(8):1766-74. 
Figures

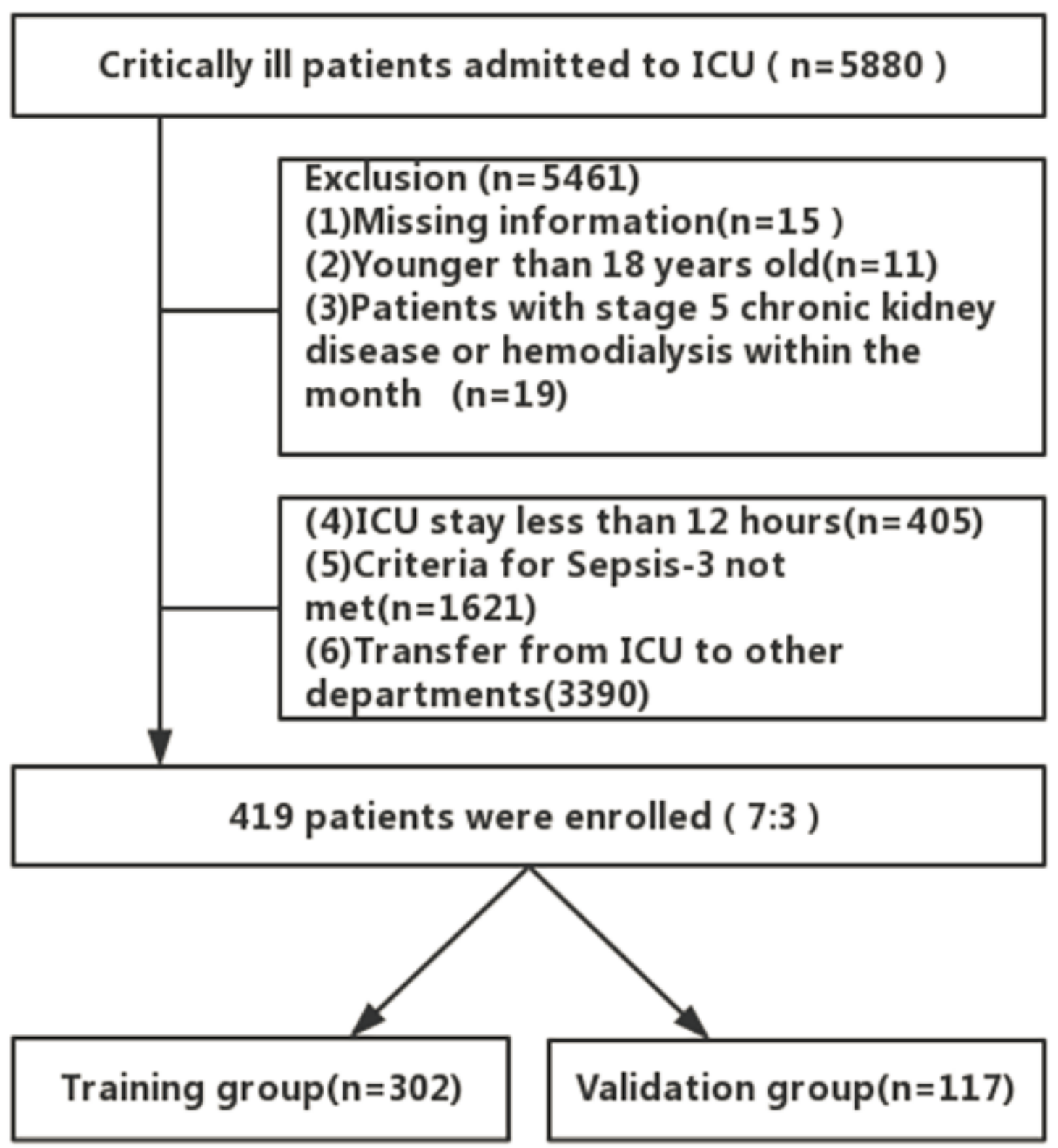

Figure 1

We selected $70 \%$ eligible patients of the sample randomly for the training set $(n=302)$ and used the remaining $30 \%$ of patients for the validation set $(n=117)$. 

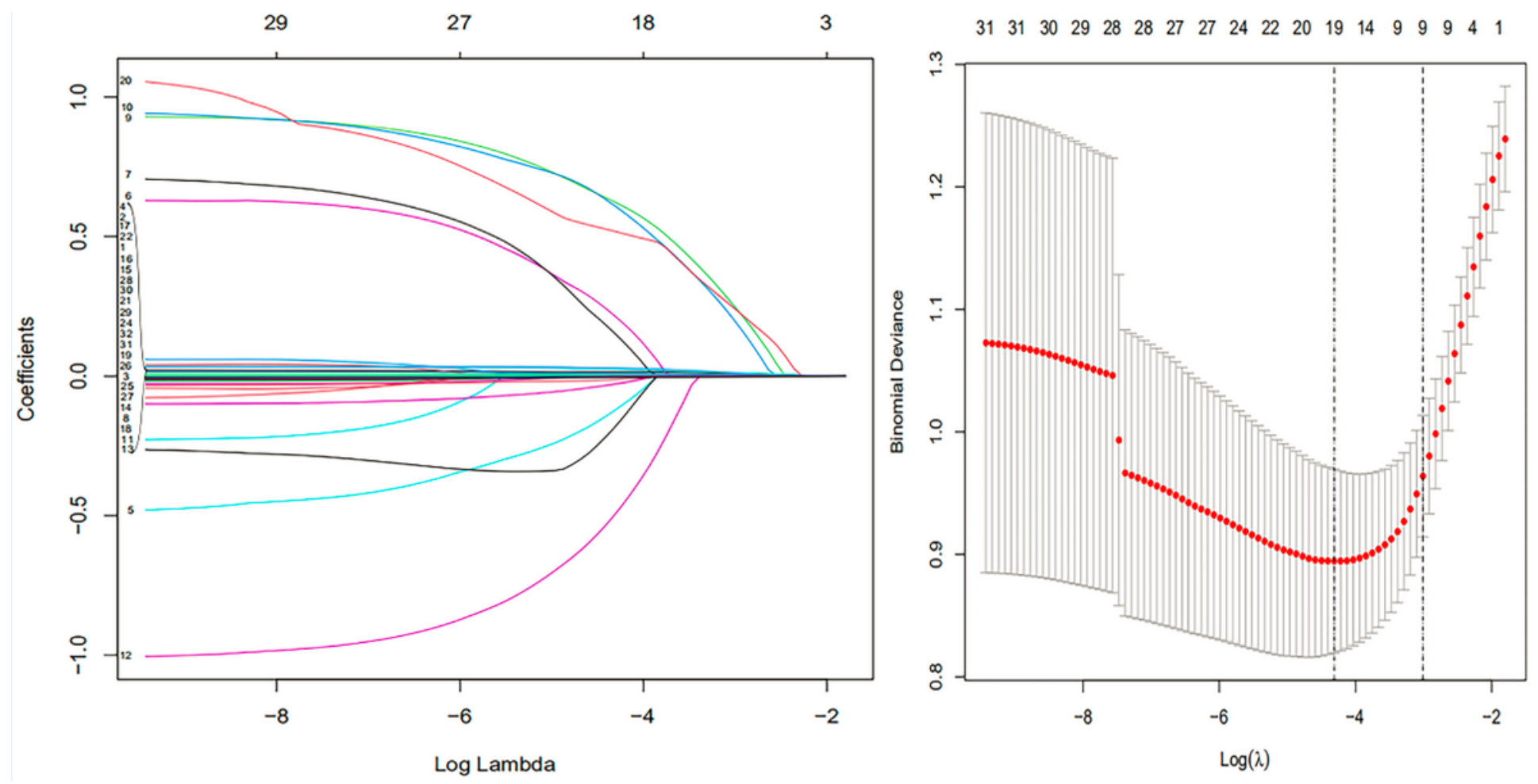

Figure 2

LASSO coefficient profiles of variables. LASSO avoid overfitting to the study data by applying a penalization/shrinkage process. This process consists of constraining the sum of the absolute values of the regression coefficients, which forces the coefficients of unimportant predictors to zero while coefficients of important predictors are regularized upward. The figure illustrates the selection process of LASSO. The abscissa stands for the step-by-step selection of variables. Eighteen variables were admitted to the logistic regression model based on the minimum average square error (ASE). 
Points

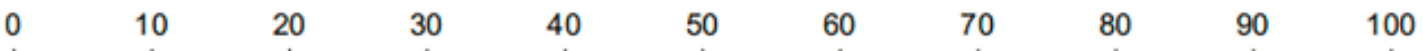

HT

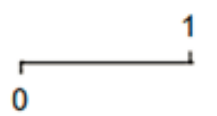

DM

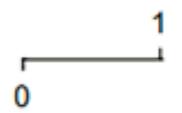

CRP

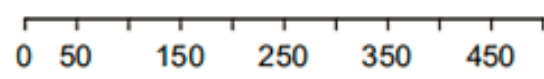

PCT

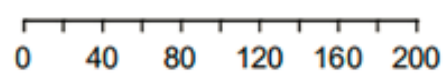

APTT

\begin{tabular}{|llllllll|}
\hline 0 & 20 & 40 & 60 & 80 & 1 & 1 & 1 \\
\hline
\end{tabular}

PLT

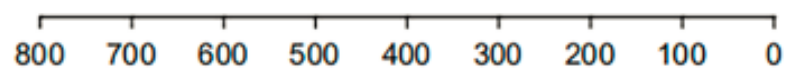

Total Points

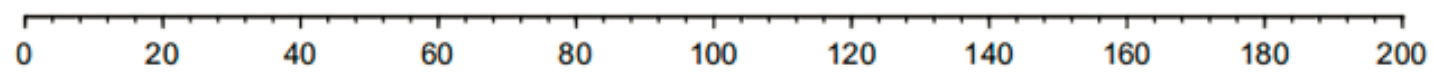

Risk of AKI

\begin{tabular}{llllllllll}
\hline & 0.05 & 0.1 & 0.2 & 0.4 & 0.6 & 0.8 & 0.9 & 0.95
\end{tabular}

\section{Figure 3}

Nomogram for the prediction of AKI. First row: point assignment of the variables; second to seventh rows: predictors of SA-AKI; eightth row: total score of seven predictors; ninth row: prediction of the risk of SAAKI. Each selected variable is represented by a vertical line. According to the value, each variable receives a point. Total points are added for each variable and matched with the probability of AKI occurrence. 


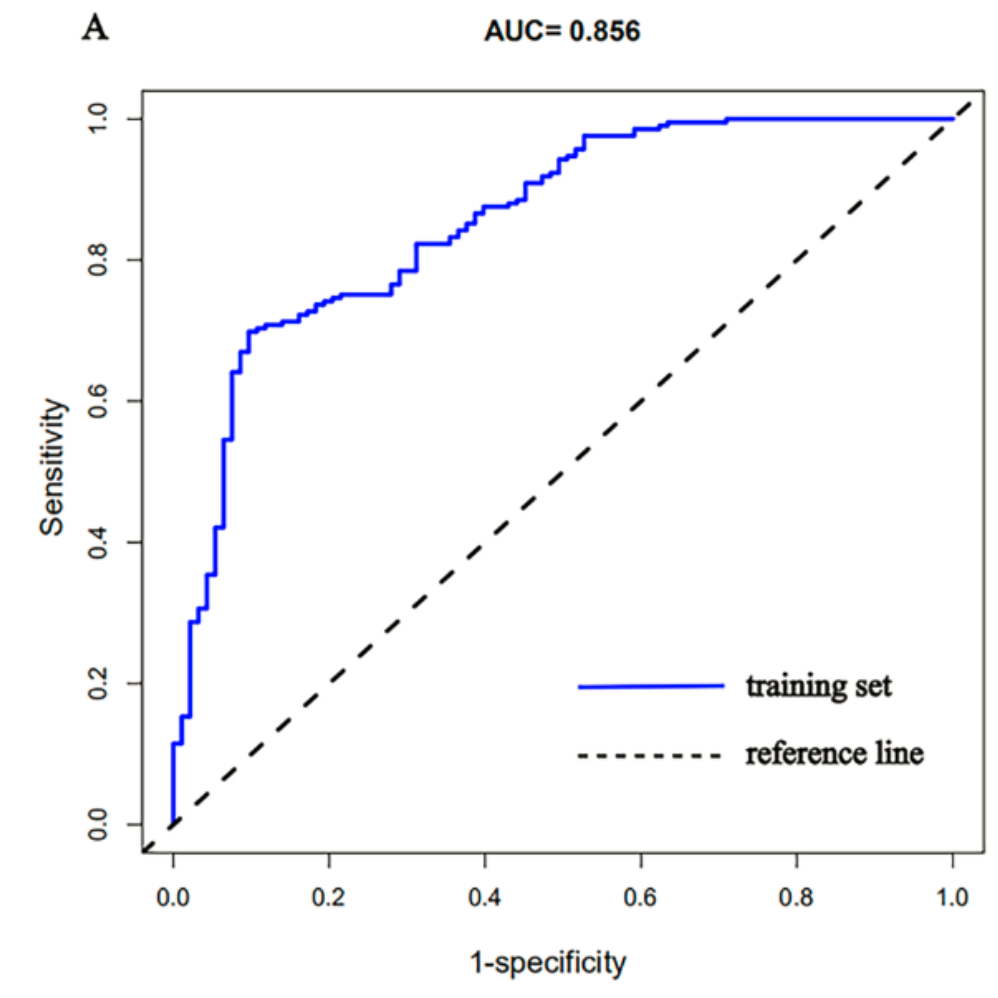

B $\quad$ AUC $=0.885$

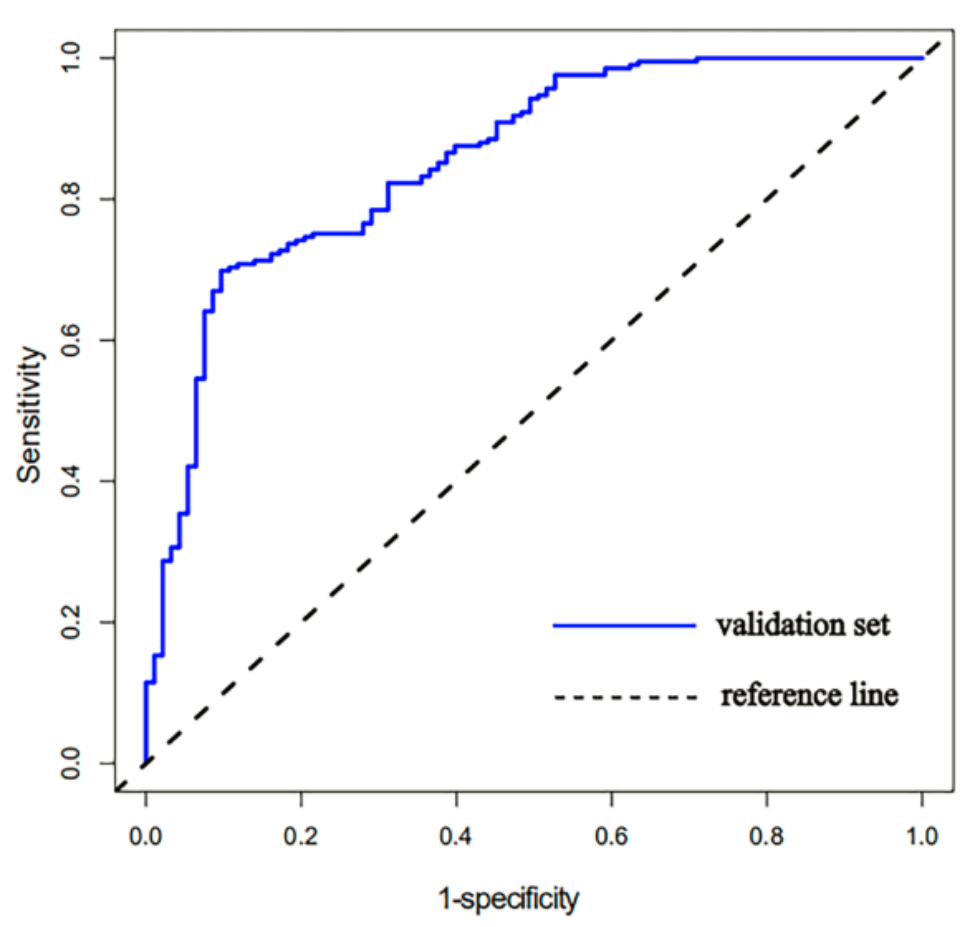

Figure 4

The ROC curves of the nomogram for the prediction of the possibility of SA-AKI. The AUC were 0.856 (95\% Cl: 0.810-0.902) in development cohort (A) and 0.885 (95\% Cl: 0.822-0.947) in validation cohort (B). The $x$-axis indicates 1 -specificity and $y$-axis the sensitivity. 


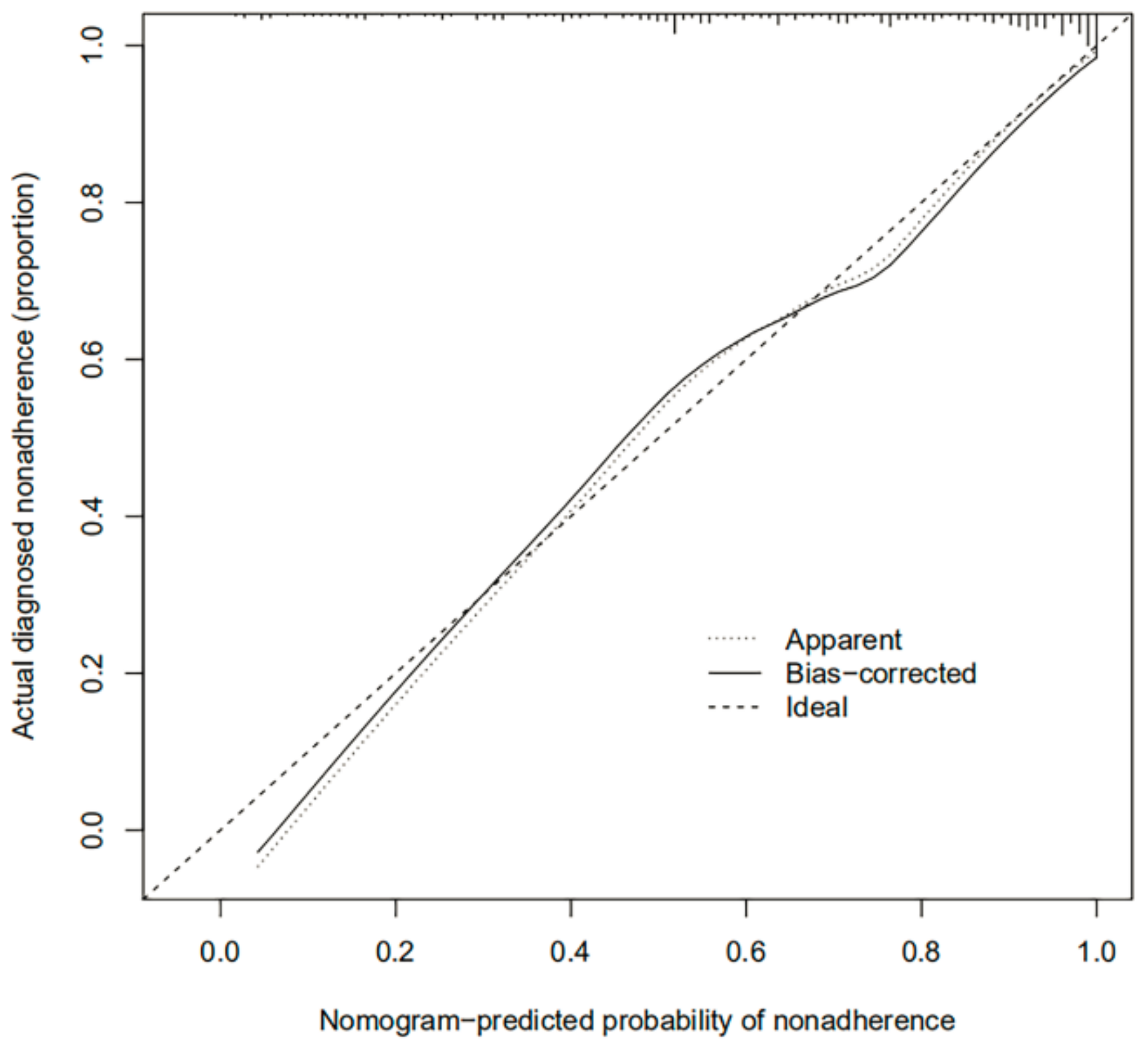

Figure 5

Calibration plot showed excellent accordance between the nomogram prediction and the actual observations of SA-AKI. Evaluation of the predictive performance for estimating the risk of SA-AKI of the nomogram.The good performance of the model can also be confirmed by the Hosmer-Lemeshow test ( $P$ value $=0.145$ ). 


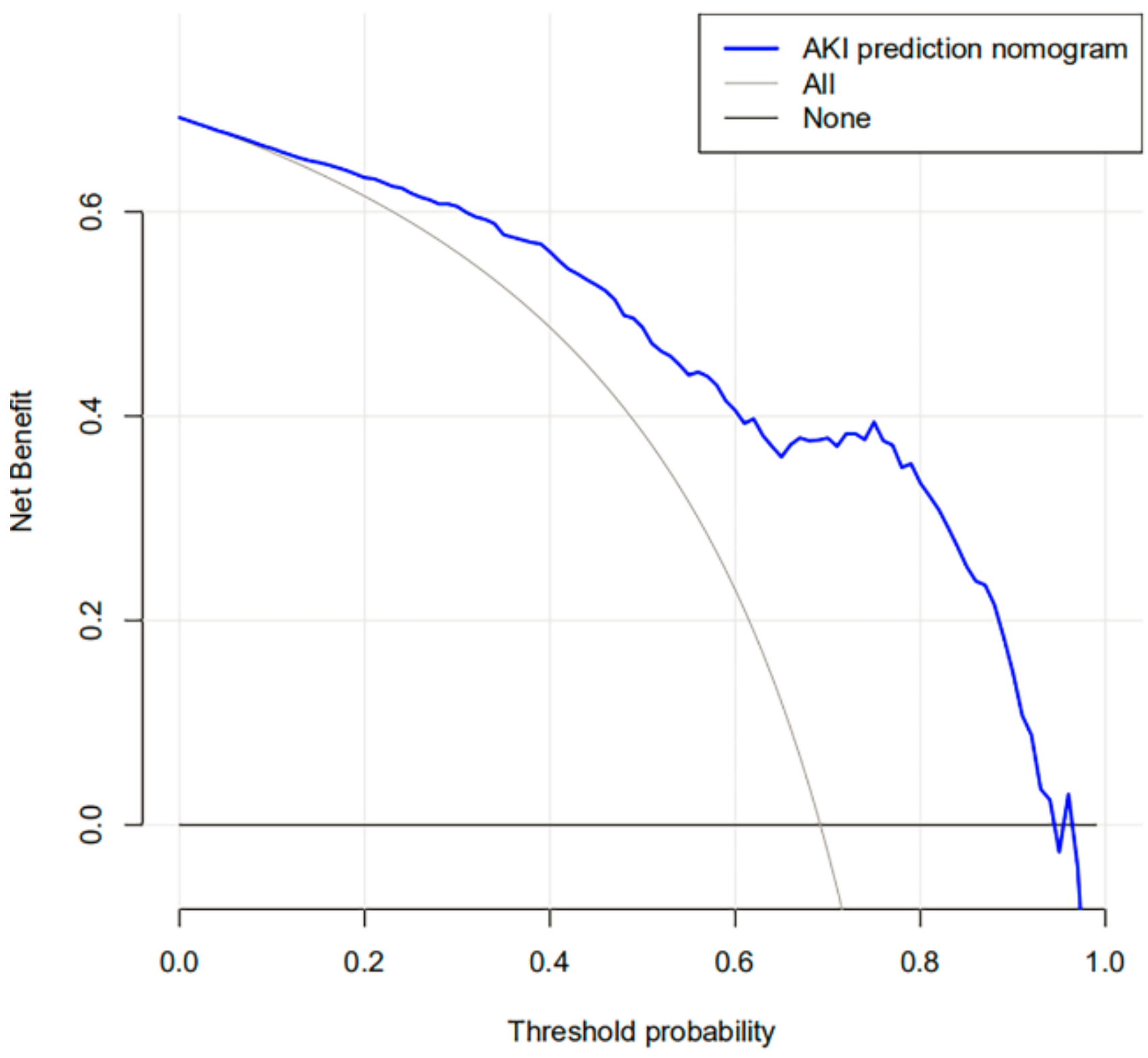

Figure 6

Decision curve analyses demonstrating the net benefit associated with the use of the nomogram-derived probability for the prediction of SA-AKI. 